\title{
Variational Laplacians for semidiscrete surfaces
}

\author{
Wolfgang Carl ${ }^{1} \cdot$ Johannes Wallner $^{1}$
}

Received: 17 December 2014 / Accepted: 23 June 2016 /

Published online: 8 July 2016

(C) The Author(s) 2016. This article is published with open access at Springerlink.com

\begin{abstract}
We study a Laplace operator on semidiscrete surfaces that is defined by variation of the Dirichlet energy functional. We show existence and its relation to the mean curvature normal, which is itself defined via variation of area. We establish several core properties like linear precision (closely related to the mean curvature of flat surfaces), and pointwise convergence. It is interesting to observe how a certain freedom in choosing area measures yields different kinds of Laplacians: it turns out that using as a measure a simple numerical integration rule yields a Laplacian previously studied as the pointwise limit of geometrically meaningful Laplacians on polygonal meshes.
\end{abstract}

Keywords Semidiscrete surface · Variational Laplacian · Mean curvature normal · Consistency

Mathematics Subject Classification (2000) 53A05 · 58E30 - 49Q20 - 41A25

Communicated by: Helmut Pottmann

Wolfgang Carl

carl@tugraz.at

Johannes Wallner

j.wallner@tugraz.at

1 Institute of Geometry, Graz University of Technology, Kopernikusgasse 24, 8010, Graz, Austria 


\section{Introduction and preliminaries}

\subsection{Introduction}

The Laplace-Beltrami operator $\Delta=-$ div o grad on smooth surfaces and Riemannian manifolds is an extremely well investigated differential operator, which plays an essential role in many fields including applications. A main strength lies in Riemannian geometry, but it is also relevant to the elementary differential geometry of surfaces in three-dimensional space, e.g. via the equation $\Delta \mathrm{id}=-2 H \mathbf{n}$ that relates the Laplacian to the mean curvature and unit normal vector field. Its intrinsic nature makes it very useful for computational applications in geometry processing, see e.g. [16], and it has therefore been extensively discretized. Discrete Laplace operators defined on triangulations share characteristics with graph Laplacians, but ideally maintain as many of the core properties of the original Laplace-Beltrami operator as possible. For contributions to this topic see e.g. [1-3, 11, 12]. Another important aspect of discretizations is a suitable convergence behavior, see e.g. [18, 20, 21].

A powerful tool to derive Laplace operators on more general surfaces arises from the calculus of variations. The Laplacian of Riemannian geometry can be seen as gradient of the Dirichlet energy, which leads to the famous "cotangent formula" Laplacian on triangle meshes, see e.g. $[8,12]$. The variational approach is also particularly suited to study the mean curvature normal $\mathbf{H}=H \mathbf{n}$, which has an interpretation as the gradient vector field of the area functional.

In this paper we follow the variational approach. Our aim is to define meaningful Laplacians on semidiscrete parametric surfaces, which are represented by a point depending on one continuous and one discrete variable. The reader is reminded that semidiscrete objects occur in the classical theory of transformations of surfaces. For a systematic and unified treatment of continuous and semidiscrete surfaces as limits of a discrete master theory we refer to the textbook [4]. The lowest-dimensional case, i.e., 2-dimensional surfaces, has been investigated from various viewpoints. The semidiscrete incarnation of conjugate surfaces is studied by [13] where piecewise-developable surfaces (including circular and conical semidiscrete surfaces) are considered from the computational viewpoint. Curvatures, in analogy to polyhedral surfaces, are the topic of [10]. Asymptotic surfaces and especially K-surfaces are investigated by [17]. The present paper however, is not concerned with any special class of semidiscrete surfaces.

\subsubsection{Outline and results}

In Section 2 we define a Laplace operator on semidiscrete surfaces by a variational principle, namely as gradient of an appropriate Dirichlet energy functional. We show that this gradient exists and provide a closed-form expression for the semidiscrete Laplacian in Theorem 1. It turns out that there is quite some freedom in the choice of the particular $L^{2}$ space which is basic to the concepts of both gradient and Dirichlet energy. Section 3 investigates the gradient of the area functional to gain a semidiscrete mean curvature normal, and establishes the relation $\Delta \mathrm{id}=-2 \mathbf{H}$ for the semidiscrete case (Theorem 2), which in turn implies that linear functions on flat surfaces 
are in the kernel of the Laplacian (i.e., the linear precision property). Section 4 discusses further properties like locality, symmetry, positive semidefiniteness, and lack of a maximum principle. The last section deals with pointwise convergence of the semidiscrete Laplacian towards the Laplace-Beltrami operator on smooth surfaces (Theorem 3).

\subsection{Variational properties of the Laplacian}

The Laplace-Beltrami operator $\Delta_{\mathcal{M}}$ on a Riemannian manifold $\mathcal{M}$ can be defined via the Dirichlet energy functional

$$
E(u)=\frac{1}{2} \int_{\mathcal{M}}\|\nabla u\|^{2} d V, \quad u \in C^{2}(\mathcal{M}, \mathbb{R}) .
$$

It is then given as the gradient of the Dirichlet energy,

$$
\Delta_{\mathcal{M}}=\nabla E,
$$

which means that for smooth test functions $u$, and all smooth 1-parameter variations $u_{\xi}$ of $u$, with the property that $\left.\frac{\partial u_{\xi}}{\partial \xi}\right|_{\xi=0}$ is compactly supported, we have

$$
\left.\frac{d}{d \xi} E\left(u_{\xi}\right)\right|_{\xi=0}=\left\langle\Delta_{\mathcal{M}} u,\left.\frac{\partial u_{\xi}}{\partial \xi}\right|_{\xi=0}\right\rangle_{L^{2}}
$$

(with the usual definition $\langle f, g\rangle_{L^{2}}=\int_{\mathcal{M}} f(x) g(x) d V(x)$; see [9, pp. 89-94]). This relation is basic to the generalization of the Laplace-Beltrami operator to discrete surfaces and will also be used in the present paper. Recall that for a surface $\mathcal{M}$ embedded in $\mathbb{R}^{3}$, the Laplace operator has a remarkable connection to the mean curvature normal. Applying the Laplacian component-wise to the identity mapping $\operatorname{id}_{\mathcal{M}}$, we get

$$
\Delta_{\mathcal{M}} \operatorname{id}_{\mathcal{M}}=-2 \mathbf{H}
$$

(see [7, p. 22]), where the mean curvature normal $\mathbf{H}=H \mathbf{n}$ is a unit normal vector $\mathbf{n}$ on $\mathcal{M}$ scaled by the corresponding mean curvature $H$. Observe that $\mathbf{H}$ is independent of the particular choice of $\mathbf{n}$, as the sign of $H$ depends on the direction of $\mathbf{n}$. This vector field likewise has a variational definition, namely

$$
-2 \mathbf{H}=\operatorname{\nabla area}(\mathcal{M}) \text {, i.e., }\left.\quad \frac{d}{d \xi} \operatorname{area}\left(p_{\xi}(\mathcal{M})\right)\right|_{\xi=0}=\left\langle-2 \mathbf{H},\left.\frac{\partial p_{\xi}}{\partial \xi}\right|_{\xi=0}\right\rangle_{L^{2}\left(\mathcal{M}, \mathbb{R}^{3}\right)}
$$

for every smooth 1-parameter variation $p_{\xi}: \mathcal{M} \rightarrow \mathbb{R}^{3}$ with $p_{0}=\mathrm{id}_{\mathcal{M}}$ (see [7, p. 7]). Here, $\operatorname{area}(\mathcal{M})=\int_{\mathcal{M}} 1 d V$ and $\langle f, g\rangle_{L^{2}\left(\mathcal{M}, \mathbb{R}^{3}\right)}=\int_{\mathcal{M}}\langle f(x), g(x)\rangle d V(x)$.

\subsection{Semidiscrete surfaces}

The semidiscrete surfaces which constitute our object of study are mappings of the form

$$
x: U \rightarrow V:(k, t) \rightarrow x(k, t), \quad \text { with } U \subset \mathbb{Z} \times \mathbb{R} \text { open, }
$$


and where $V$ is a vector space equipped with a positive definite scalar product $\langle\cdot, \cdot\rangle_{V}$. Throughout this paper we assume that $x$ is at least twice continuously differentiable in the second argument, and we denote the corresponding set of mappings by $C_{\text {sd }}^{2}(U, V)$. Accordingly, the set of semidiscrete functions that are merely continuous in the second argument is denoted by $C_{\mathrm{sd}}(U, V)$. With the help of the canonical hat function $\varphi(s):=\max \{1-|s|, 0\}$, we extend $x$ to a mapping, again called $x$,

$$
x: \widehat{U} \rightarrow V:(s, t) \mapsto \sum_{k:(k, t) \in U} \varphi(s-k) x(k, t),
$$

where the domain $\widehat{U}$ is constructed as a disjoint union of strips $U_{k} \subset \mathbb{R}^{2}$, each strip being defined as

$$
U_{k}:=\bigcup_{t:(k, t) \in U \wedge(k+1, t) \in U}[k, k+1] \times\{t\} .
$$

In the non-degenerate case, this procedure converts a sequence of curves into a piecewise-ruled surface, connecting corresponding points $x(k, t)$ and $x(k+1, t)$ by straight line segments. For each pair of successive curves $x(k, \cdot)$ and $x(k+1, \cdot)$ there is a ruled surface strip, which is treated separately from the others as far as the domain of definition is concerned. This procedure does not alter the values $x(s, t)$ where $s$ happens to equal an integer $k \in \mathbb{Z} ; x(s, t)$ has the same value regardless of the question if $s$ is considered as an element of $[k-1, k]$ or as an element of $[k, k+1]$. We call the procedure of converting a semidiscrete surface $x(k, t)$ to a piecewise-ruled surface $x(s, t)$ an "extension", even if $U$ is not a subset of $\widehat{U}$.

In order to make the upcoming formulas shorter and thus better readable, we set up the following notation. For the derivatives of $x(k, t)$ with respect to the variable $t$, we write $x^{\prime}, x^{\prime \prime}$, and so forth. Finite differences in the discrete direction are denoted by

$$
\delta x(s, t):=x(k+1, t)-x(k, t), \quad \text { for } s \in[k, k+1] .
$$

Note that in contrast to $x$ itself, the discrete derivative $\delta x$ does have different values for $s=k \in \mathbb{Z}$, depending on whether $s$ is thought to be contained in $[k-1, k]$ or in $[k, k+1]$. We resolve this ambiguity by always making it clear which of the two corresponding surface strips we are considering.

We call a semidiscrete surface regular, if all its surface strips are regular in the usual sense, i.e., if

$$
\left\{\delta x(s, t), x^{\prime}(s, t)\right\}, \quad s \in[k, k+1]
$$

is linearly independent throughout. Moreover, we call $(k, t) \in U$ an inner point, if

$$
\{k-1, k, k+1\} \times(t-\varepsilon, t+\varepsilon) \subset U,
$$

for some $\varepsilon>0$. Otherwise it is called a boundary point. The set of inner points of $U$ will be denoted by $U^{\text {inn }}$.

Note that we do not make any assumptions on the embeddedness of the surfaces we study. Later, when considering a real-valued function " $u$ " on a semidiscrete surface $x$, we regard it as defined in $U$ rather than in $x(U)$. Such a function $u$ therefore formally is a semidiscrete surface in its own right and we use the same notation as for the surface $x$. We call $u$ smooth, if it is at least twice continuously differentiable in the second argument, i.e., if $u \in C_{\mathrm{sd}}^{2}(U, \mathbb{R})$. 
Remark 1 It is easy to see that a semidiscrete surface $x(s, t)$ is regular for all $s \in$ $[k, k+1]$ if $x^{\prime}(k, t), x^{\prime}(k+1, t)$ and $\delta x(k, t)$ are linearly independent (in which case the ruled surface strip corresponding to $s \in[k, k+1]$ is a regular skew ruled surface). In case those vectors are linearly dependent, regularity in that interval is equivalent to $\left|\delta x(k, t), x^{\prime}(k, t)\right| \cdot\left|\delta x(k, t), x^{\prime}(k+1, t)\right|>0$, for any determinant form $|\cdot, \cdot|$ on a plane containing these three vectors (the strip then has a torsal generator whose singular point $x\left(s^{*}, t\right)$ obeys $s^{*} \notin[k, k+1]$; cf. [14, Section 5.1.1]).

\section{Variational definition of a semidiscrete Laplace operator}

This section aims at a meaningful definition of a Laplace operator on semidiscrete surfaces. Mimicking the smooth case, we define a semidiscrete Laplacian as gradient of an appropriate Dirichlet energy functional. For this purpose we first discuss area measures.

\subsection{Integration and Laplacian on semidiscrete surfaces}

Consider a semidiscrete surface $x$ with open domain $U \subset \mathbb{Z} \times \mathbb{R}$, which has been extended to a piecewise-ruled surface defined in the domain $\widehat{U}$, as described above (cf. Eq. 1).

A reasonable definition of its area obviously is given by the sum of the areas of individual ruled surface strips, which in terms of the matrix I of the first fundamental form is expressed as

$$
\operatorname{area}(x)=\iint_{\widehat{U}} \sqrt{\operatorname{det} \mathrm{I}(s, t)} d s d t, \quad \text { with } \mathrm{I}=\left(\begin{array}{cc}
\|\delta x\|^{2} & \left\langle\delta x, x^{\prime}\right\rangle \\
\left\langle\delta x, x^{\prime}\right\rangle & \left\|x^{\prime}\right\|^{2}
\end{array}\right)
$$

(see [6, p. 98]). Note that, in order to resolve the ambiguity in the definition of $\delta x$, the double integral over $\widehat{U}$ has to be interpreted as the sum of double integrals over the individual strips $U_{k}$ stated in Eq. 2.

It makes sense to generalize this definition by replacing Lebesgue measure $d s d t$ by other measures. We start with a Borel measure $\mu_{0}$ supported on the unit interval $[0,1]$, whose zeroth and first moments have the following values:

$$
m_{0}=\int_{[0,1]} 1 d \mu_{0}(s)=1 \quad \text { and } \quad m_{1}=\int_{[0,1]} s d \mu_{0}(s)=\frac{1}{2} .
$$

That is, we require integration of polynomials up to degree 1 to coincide with integration w.r.t. Lebesgue measure. A stronger property is symmetry of the measure, meaning that

$$
\int_{[0,1]} f(s) d \mu_{0}(s)=\int_{[0,1]} f(1-s) d \mu_{0}(s), \quad \text { for all } f \in L^{1}\left([0,1], \mu_{0}\right) .
$$

Together with $m_{0}=1$ symmetry implies $m_{1}=1 / 2$. This symmetry property is not required except in Theorem 3 , where it is explicitly mentioned.

We will see that these assumptions are crucial for some important properties of the Laplacian, and also for convergence. We actually construct an entire family of 
semidiscrete Laplace operators, depending on the type of integration we employ. Note that in particular the measure $\mu_{0}$ might be a numerical integration rule, like the midpoint rule or the trapezoidal rule. As it turns out, a particular choice of measure leads to the semidiscrete Laplacian introduced in [5] as a pointwise limit of the discrete construction of Alexa and Wardetzky [1]. We discuss this connection in Section 2.2.

Now, by translation, $\mu_{0}$ acts as a measure on each interval $[k, k+1]$, and we denote the sum of measures on the disjoint union of intervals $[k, k+1]$ by $\mu$. With the Lebesgue measure $\lambda$ on the reals, we consider the product measure $\mu \otimes \lambda$ on the disjoint union of strips $[k, k+1] \times \mathbb{R}$. It is precisely this measure which we use for integration in the domain $\widehat{U}$ :

Definition 1 Consider a semidiscrete surface $x: U \rightarrow V$, extended to a piecewiseruled surface $x: \widehat{U} \rightarrow V$. We use its first fundamental form I and the measure $\mu \otimes \lambda$ on $\widehat{U}$ to define the surface integral of a function $u: \widehat{U} \rightarrow \mathbb{R}:$

$$
\int_{x} u d A:=\int_{\widehat{U}} u(s, t) \sqrt{\operatorname{det} \mathrm{I}(s, t)} d(\mu \otimes \lambda)(s, t) .
$$

The surface area is given by $\operatorname{area}_{\mu}(x):=\int_{x} 1 d A$.

Again, by the integral over $\widehat{U}$ we mean the sum of integrals over the individual strips $U_{k}$ given by Eq. 2 . Note that for nonnegative functions $u$, the integral always exists and $\int_{x} u d A \in[0, \infty]$, whereas for general $u, \int_{x} u d A=\int_{x} \max (u, 0) d A-$ $\int_{x} \max (-u, 0) d A$ is only defined if at least one of the involved expressions is finite.

Example 1 This definition in particular applies to a semidiscrete function $u: U \rightarrow$ $\mathbb{R}$, which has been extended to a piecewise-linear function $u: \widehat{U} \rightarrow \mathbb{R}$ by linear interpolation:

$$
u(s, t)=\sum_{k:(k, t) \in U} \varphi(s-k) u(k, t) .
$$

If $u$ vanishes at the boundary of $U$, we can write its surface integral as

$$
\int_{x} u d A=\sum_{k \in \mathbb{Z}} \int_{\pi_{2}\left(U_{k}\right)} u(k, t) \mathbf{a}(k, t) d \lambda(t),
$$

where $\pi_{2}: \mathbb{R} \times \mathbb{R} \rightarrow \mathbb{R}:(s, t) \mapsto t$, and the semidiscrete function $\mathbf{a}$ is defined by

$$
\mathbf{a}(k, t):=\int_{[k-1, k] \sqcup[k, k+1]} \varphi(s-k) \sqrt{\operatorname{det} \mathrm{I}(s, t)} d \mu(s) .
$$

Here, the integral over $[k-1, k] \sqcup[k, k+1]$ represents the sum of the integrals over the intervals $[k-1, k]$ and $[k, k+1]$. This formula follows from computing the left hand side by the iterated integral $\sum_{k} \int_{\pi_{2}\left(U_{k}\right)}\left(\int_{[k, k+1]} u \sqrt{\operatorname{det} \mathrm{I}} d \mu(s)\right) d \lambda(t)$ and using $u(s, t)=\sum_{j} \varphi(s-j) u(j, t)$ to express the interior integral as $u(k, t) \int_{[k, k+1]} \varphi(s-$ $k) \sqrt{\operatorname{det} \mathrm{I}} d \mu(s)+u(k+1, t) \int_{[k, k+1]} \varphi(s-k-1) \sqrt{\operatorname{det} \mathrm{I}} d \mu(s)$. An index shift yields the formula given above. 
Definition 2 Given a semidiscrete surface $x$ with domain $U$, we define $L^{2}$ inner products for semidiscrete real-valued (resp. $V$-valued) functions $u, v$ with the same domain by letting

$$
\langle u, v\rangle_{L^{2}(x)}:=\int_{x} u v d A, \quad \text { resp. } \quad\langle u, v\rangle_{L^{2}(x, V)}:=\int_{x}\langle u, v\rangle_{V} d A .
$$

The integrals in the previous formulas mean that the semidiscrete functions $u, v$ are multiplied to create a semidiscrete product function $(u \cdot v)(k, t)\left(\operatorname{resp} .\langle u, v\rangle_{V}(k, t)\right)$, which for the purpose of integration undergoes linear interpolation. The inner products are, for instance, well defined for semidiscrete functions that are continuous in the second argument and have finite $L^{2}$ norm.

For the Dirichlet energy of a semidiscrete function we use the following definition:

Definition 3 Let $x$ be a regular semidiscrete surface defined on $U$ and let $u, v$ : $U \rightarrow \mathbb{R}$ be smooth semidiscrete functions, considered as functions on $x$. Then we use the extended functions $u(s, t), v(s, t)$ over the extended surface $x(s, t)$ to define the Dirichlet energy $E_{\mu}(u)$ and the corresponding symmetric bilinear form $\mathcal{E}_{\mu}(u, v)$ via

$$
E_{\mu}(u):=\frac{1}{2} \int_{x}\|\nabla u\|^{2} d A, \quad \mathcal{E}_{\mu}(u, v):=\frac{1}{2} \int_{x}\langle\nabla u, \nabla v\rangle d A .
$$

As to the gradients of real-valued functions $u(s, t), v(s, t)$ on a parametric surface $x(s, t)$, recall that $\langle\nabla u, \nabla v\rangle=\left(\begin{array}{c}\partial u / \partial s \\ \partial u / \partial t\end{array}\right)^{T} \cdot \mathrm{I}^{-1} \cdot\left(\begin{array}{c}\partial v / \partial s \\ \partial v / \partial t\end{array}\right)$, where $\mathrm{I}(s, t)$ is the matrix of the first fundamental form. This leads to the following explicit expression for the Dirichlet energy:

$$
E_{\mu}(u)=\frac{1}{2} \int_{\widehat{U}} \operatorname{det} \mathrm{I}^{-1 / 2}\left(\left\|x^{\prime}\right\|^{2}(\delta u)^{2}-2\left\langle\delta x, x^{\prime}\right\rangle(\delta u)\left(u^{\prime}\right)+\|\delta x\|^{2}\left(u^{\prime}\right)^{2}\right) d(\mu \otimes \lambda) .
$$

Observe that $E_{\mu}(u)$ essentially is the ordinary Dirichlet energy of the piecewisesmooth function $u(s, t)$ over the piecewise-smooth surface $x(s, t)$.

It is tempting to employ $L^{2}$ notation for the definition of the Dirichlet energy. We will not do that, since the integrand is not generated by extending a semidiscrete function, and therefore does not fit Definition 2.

Next, we generalize the notion of the gradient of an energy functional to the semidiscrete case. For that we consider "admissible" variations of semidiscrete functions:

Definition 4 An admissible variation $x_{\xi}(k, t)$ of a smooth semidiscrete mapping $x$ : $U \rightarrow V$ is a $V$-valued function of arguments $(\xi, k, t) \in(-\varepsilon, \varepsilon) \times U$, which depends smoothly on $\xi$ and $t$, coincides with $x(k, t)$ for $\xi=0$, and such that $x_{\xi}(k, t)$ does not depend on $\xi$ outside a compact subset $K$ of $U^{\text {inn }}$. Moreover, we call a subset $B \subset U$ associated with the variation $x_{\xi}$, if it is open and bounded, with $K \subset B \subset \bar{B} \subset U$ and $(k, t) \in K \Longrightarrow(k-1, t),(k+1, t) \in B$. For the derivative of $x_{\xi}$ with respect to $\xi$ we use the notation

$$
\dot{x}(k, t):=\left.\frac{\partial}{\partial \xi} x_{\xi}(k, t)\right|_{\xi=0} .
$$


This definition in particular applies to admissible variations $u_{\xi}(k, t)$ of smooth semidiscrete functions $u: U \rightarrow \mathbb{R}$. In what follows we discuss the variation of energy, and the variation of surface area, even if these quantities are not finite. As admissible variations only take effect on compact parts of the domain $U$, the corresponding change in energy or surface area can be defined in a meaningful way.

Definition 5 Let $x: U \rightarrow V$ be a regular semidiscrete surface and let $E$ be a functional on $C_{\mathrm{sd}}^{2}(U, \mathbb{R})$, with the property that there exists an operator $\nabla E$ : $C_{\text {sd }}^{2}(U, \mathbb{R}) \rightarrow C_{\text {sd }}\left(U^{\text {inn }}, \mathbb{R}\right)$, such that for every $u \in C_{\text {sd }}^{2}(U, \mathbb{R})$ and all admissible 1-parameter variations $u_{\xi}$ of $u$ with associated subset $B \subset U$, we have

$$
\left.\frac{d}{d \xi} E\left(\left.u_{\xi}\right|_{B}\right)\right|_{\xi=0}=\langle\nabla E(u), \dot{u}\rangle_{L^{2}(x)} .
$$

Then $\nabla E$ is called the gradient of $E$. In particular, we define the semidiscrete Laplace operator $\Delta_{\mathrm{sd}}$ on $x$ as the gradient of the Dirichlet energy functional $E_{\mu}$, i.e.,

$$
\Delta_{\mathrm{sd}}:=\nabla E_{\mu} .
$$

Note that this definition is independent of the particular choice of the bounded open subset $B \subset U$ associated with a variation $u_{\xi}$ of $u$.

Theorem 1 If $x: U \rightarrow V$ is a regular semidiscrete surface, then the semidiscrete Laplacian $\Delta_{\mathrm{sd}} u$ exists for all smooth semidiscrete functions $u$ defined in the same domain:

$$
\left(\Delta_{\mathrm{sd}} u\right)(k, t)=\frac{1}{\mathbf{a}(k, t)}\left(\delta \mathbf{b}(k, t)+\mathbf{c}^{\prime}(k, t)\right),
$$

with $\mathbf{a}$ as in Example 1, and with semidiscrete functions $\mathbf{b}$, c defined by

$$
\begin{aligned}
\mathbf{b}(k, \cdot) & :=\int_{[k-1, k]} \operatorname{det} \mathrm{I}^{-1 / 2}\left(\left\langle\delta x, x^{\prime}\right\rangle u^{\prime}-\left\|x^{\prime}\right\|^{2} \delta u\right) d \mu, \\
\mathbf{c}(k, \cdot) & :=\int_{[k-1, k] \sqcup[k, k+1]} \operatorname{det} \mathrm{I}^{-1 / 2} \varphi(s-k)\left(\left\langle\delta x, x^{\prime}\right\rangle \delta u-\|\delta x\|^{2} u^{\prime}\right) d \mu .
\end{aligned}
$$

Proof Let $u_{\xi}$ be an admissible variation of $u$ with derivative $\dot{u}$ and let $B \subset U$ be associated with $u_{\xi}$. We compute the derivative of the Dirichlet energy by using the Leibniz rule (which applies because all occurring functions are smooth in the variables $\xi$ and $t$, and $\dot{u}$ has compact support):

$$
\begin{aligned}
\left.\frac{d}{d \xi} E_{\mu}\left(\left.u_{\xi}\right|_{B}\right)\right|_{\xi=0}= & \int_{\widehat{U}} \operatorname{det} \mathrm{I}^{-1 / 2}\left(\left\|x^{\prime}\right\|^{2} \delta u \delta \dot{u}-\left\langle\delta x, x^{\prime}\right\rangle\left(\delta u \dot{u}^{\prime}+u^{\prime} \delta \dot{u}\right)+\|\delta x\|^{2} u^{\prime} \dot{u}^{\prime}\right) \\
& \times d(\mu \otimes \lambda) \\
= & \int_{\widehat{U}}\left(-b(s, t) \delta \dot{u}(s, t)-c(s, t) \dot{u}^{\prime}(s, t)\right) d(\mu \otimes \lambda)(s, t), \text { where } \\
b: & \operatorname{det}^{-1 / 2}\left(\left\langle\delta x, x^{\prime}\right\rangle u^{\prime}-\left\|x^{\prime}\right\|^{2} \delta u\right), \\
c & :=\operatorname{det} \mathrm{I}^{-1 / 2}\left(\left\langle\delta x, x^{\prime}\right\rangle \delta u-\|\delta x\|^{2} u^{\prime}\right) .
\end{aligned}
$$


Next we apply integration by parts w.r.t. $t$ to the second summand:

$$
\begin{aligned}
\left.\frac{d}{d \xi} E_{\mu}\left(\left.u_{\xi}\right|_{B}\right)\right|_{\xi=0}= & \int_{\widehat{U}}\left(-b(s, t) \delta \dot{u}(s, t)+c^{\prime}(s, t) \dot{u}(s, t)\right) d(\mu \otimes \lambda)(s, t) \\
= & \sum_{k \in \mathbb{Z}} \int_{\pi_{2}\left(U_{k}\right)} \int_{s \in[k, k+1]} b(s, t)(\dot{u}(k, t)-\dot{u}(k+1, t)) \\
& +c^{\prime}(s, t)(\varphi(s-k) \dot{u}(k, t)+\varphi(s-k-1) \dot{u}(k+1, t)) d \mu(s) d \lambda(t) .
\end{aligned}
$$

Observe that the boundary terms vanish, since the support of $\dot{u}$ is contained in $U^{\text {inn }}$. Finally, an index shift yields

$$
\left.\frac{d}{d \xi} E_{\mu}\left(\left.u_{\xi}\right|_{B}\right)\right|_{\xi=0}=\sum_{k \in \mathbb{Z}} \int_{\pi_{2}\left(U_{k}\right)}\left(\delta \mathbf{b}(k, t)+\mathbf{c}^{\prime}(k, t)\right) \dot{u}(k, t) d \lambda(t)=\left\langle\Delta_{\mathrm{sd}} u, \dot{u}\right\rangle_{L^{2}(x)},
$$

with b, c, and $\Delta_{\mathrm{sd}} u$ as stated above (cf. also Example 1).

\subsection{Example: semidiscrete Laplacians arising as limits of discrete ones}

As demonstrated in [5], the discrete Laplace operator $\mathbb{L}$ constructed by Alexa and Wardetzky [1] for functions defined on the vertices of a polygonal mesh gives rise to a Laplace operator on semidiscrete surfaces via pointwise limits. We may discretize a regular semidiscrete surface $x: U \rightarrow \mathbb{R}^{3}$ and a smooth function $u: U \rightarrow \mathbb{R}$ near a point of interest $(k, t) \in U^{\text {inn }}$ by letting

$$
x_{i j}^{\varepsilon}:=x(k+i, t+\varepsilon j), \quad u^{\varepsilon}\left(x_{i j}^{\varepsilon}\right):=u(k+i, t+\varepsilon j) .
$$

This defines the vertices $x_{i j}^{\varepsilon}$ of a quad mesh with regular combinatorics, and function values on these vertices. The discrete Lapace operator on that mesh is denoted by $\mathbb{L}^{\varepsilon}$, and we let

$$
\left(\Delta_{\lim } u\right)(k, t):=\left.\lim _{\varepsilon \rightarrow 0}\left(\mathbb{L}^{\varepsilon} u^{\varepsilon}\right)\right|_{0,0} .
$$

Existence and properties of this limit were investigated in [5], in particular independence of the limit from the still remaining degrees of freedom in the construction of $\mathbb{L}$. There is a remarkable connection between our semidiscrete Laplacian $\Delta_{\text {sd }}$ and the semidiscrete Laplacian $\Delta_{\text {lim }}$ which arises by pointwise limits. In fact, if the measure $\mu_{0}$ used to construct $\Delta_{\mathrm{sd}}$ is taken as the midpoint rule for numerical integration (i.e., $\left.\int_{[0,1]} f(s) d \mu_{0}(s)=f\left(\frac{1}{2}\right)\right)$, then they are equal:

$$
\mu_{0}\left(\left\{\frac{1}{2}\right\}\right)=1 \Longrightarrow \Delta_{\mathrm{sd}} u=\Delta_{\lim } u, \quad \forall u \in C_{\mathrm{sd}}^{2}(U, \mathbb{R}) .
$$

This claim is easily verified by comparing the formulae for $\Delta_{\lim } u$ given by [5, Corollary 1] with the explicit expressions stated in Theorem 1 of the present paper: If $\mu_{0}\left(\left\{\frac{1}{2}\right\}\right)=1$,

$$
\begin{aligned}
\mathbf{a}(k, t) & =\int_{[k-1, k] \sqcup[k, k+1]} \varphi(s-k)\left\|\delta x(s, t) \times x^{\prime}(s, t)\right\| d \mu(s) \\
& =\frac{1}{4}\left(\left\|\left(x_{1}-x\right) \times\left(x_{1}^{\prime}+x^{\prime}\right)\right\|+\left\|\left(x_{\overline{1}}-x\right) \times\left(x_{\overline{1}}^{\prime}+x^{\prime}\right)\right\|\right)=\frac{A_{1}+A_{\overline{1}}}{4},
\end{aligned}
$$


where we adopt the notation from [5, Corollary 1]. In particular, $x_{1}(k, t)=x(k+1, t)$ and $x_{\overline{1}}(k, t)=x(k-1, t)$. Likewise, we get

$$
\begin{aligned}
\mathbf{b}(k, t) & =\frac{1}{2 A_{1}}\left(\left\langle x_{1}-x, x_{1}^{\prime}+x^{\prime}\right\rangle\left(u_{1}^{\prime}+u^{\prime}\right)-\left\|x_{1}^{\prime}+x^{\prime}\right\|^{2}\left(u_{1}-u\right)\right), \quad \text { and } \\
\mathbf{c}(k, t) & =\frac{1}{2 A_{1}}\left(\left\|x_{1}-x\right\|^{2}\left(u_{1}^{\prime}+u^{\prime}\right)-\left\langle x_{1}-x, x_{1}^{\prime}+x^{\prime}\right\rangle\left(u_{1}-u\right)\right)=-\frac{\alpha_{1}}{2 A_{1}} .
\end{aligned}
$$

By inserting these functions into Eq. 6 and comparing the resulting expression with the formula stated in [5, Corollary 1], we see that $\Delta_{\mathrm{sd}} u=\Delta_{\lim } u$.

\section{Semidiscrete mean curvature normals}

Before we analyze further properties of the semidiscrete Laplace operator, we discuss its connection to the mean curvature normal. Recall from the introductory section the relations between the Laplacian and the mean curvature normal, which hold for smooth surfaces embedded in $\mathbb{R}^{3}$ : On the one hand, $\Delta_{\mathcal{M}} \mathrm{id}_{\mathcal{M}}=-2 \mathbf{H}$, on the other hand the mean curvature normal itself has the variational definition $-2 \mathbf{H}=\nabla \operatorname{area}(\mathcal{M})$. Here we consider the semidiscrete version of these objects and the relations between them. Our notation is not entirely the same as in Section 1.2, because we deal with parametric surfaces.

\subsection{Variational properties of mean curvature}

Definition 6 Let $F$ be a functional on $C_{\text {sd }}^{2}(U, V)$ and let $x: U \rightarrow V$ be a semidiscrete surface with the property that there exists a function $\nabla F(x) \in C_{\mathrm{sd}}\left(U^{\mathrm{inn}}, V\right)$, such that for all admissible 1-parameter variations $x_{\xi}$ of $x$ with associated subset $B \subset U$, we have

$$
\left.\frac{d}{d \xi} F\left(\left.x_{\xi}\right|_{B}\right)\right|_{\xi=0}=\langle\nabla F(x), \dot{x}\rangle_{L^{2}(x, V)} .
$$

Then $\nabla F(x)$ is called the gradient of $F$ at $x$. In particular, the semidiscrete mean curvature normal $\mathbf{H}_{\mathrm{sd}}$ of a regular semidiscrete surface $x$ is defined as

$$
\mathbf{H}_{\mathrm{sd}}:=-\frac{1}{2} \nabla \operatorname{area}_{\mu}(x) .
$$

Theorem 2 For regular semidiscrete surfaces $x$, the mean curvature normal vector field exists and can be computed by applying the Laplacian componentwise to the identity mapping on $x$ :

$$
\Delta_{\mathrm{sd}} x=-2 \mathbf{H}_{\mathrm{sd}}
$$

Proof Let $x_{\xi}(k, t)$ be an admissible variation of $x$. Each semidiscrete surface $x_{\xi}(k, t)$ is extended to a piecewise-ruled surface $x_{\xi}(s, t)$, having first fundamental form $\mathrm{I}_{\xi}(s, t)$ (cf. Eq. 3). By definition, $x_{\xi}(k, t)$ is independent of $\xi$ outside a compact subset $K$ of $U^{\text {inn }}$. Thus, by a standard argument, the piecewise-ruled surfaces $x_{\xi}(s, t)$ 
are regular for all $\xi$ in some interval $(-h, h)$, because $\sqrt{\operatorname{det} \mathrm{I}_{\xi}}$, i.e., the area spanned by the partial derivatives of $x_{\xi}$, is positive in a compact set $\{0\} \times K^{\prime} \subset \mathbb{R} \times \widehat{U}$, thus positive in a neighbourhood of this set, and consequently positive in a product set $(-h, h) \times K^{\prime}$.

Thus, we may compute

$$
\frac{\partial}{\partial \xi} \sqrt{\operatorname{det} I_{\xi}}=\frac{1}{2 \sqrt{\operatorname{det} I_{\xi}}} \frac{\partial}{\partial \xi}\left[\left\|\delta x_{\xi}\right\|^{2}\left\|x_{\xi}^{\prime}\right\|^{2}-\left\langle\delta x_{\xi}, x_{\xi}^{\prime}\right\rangle^{2}\right] .
$$

For $\xi=0$, this expression is simplified by computing the individual derivatives $\frac{\partial}{\partial \xi}\left\|\delta x_{\xi}\right\|^{2}=2\langle\delta x, \delta \dot{x}\rangle, \frac{\partial}{\partial \xi}\left\|x_{\xi}^{\prime}\right\|^{2}=2\left\langle x^{\prime}, \dot{x}^{\prime}\right\rangle$, and $\frac{\partial}{\partial \xi}\left\langle\delta x_{\xi}, x_{\xi}^{\prime}\right\rangle^{2}=2\left(\left\langle x^{\prime}, \delta \dot{x}\right\rangle+\right.$ $\left.\left\langle\delta x, \dot{x}^{\prime}\right\rangle\right)\left\langle\delta x, x^{\prime}\right\rangle$. We get

$$
\left.\frac{d}{d \xi} \operatorname{area}_{\mu}\left(\left.x_{\xi}\right|_{B}\right)\right|_{\xi=0}=\int_{\widehat{U}}\left(-\langle b(s, t), \delta \dot{x}(s, t)\rangle-\left\langle c(s, t), \dot{x}^{\prime}(s, t)\right\rangle\right) d(\mu \otimes \lambda)(s, t),
$$

where the functions $b(s, t)$ and $c(s, t)$ are the same as in Eq. 7, and the previous formula is the same as the expression for the derivative of the Dirichlet energy in the proof of Thm. 1, only with $x$ instead of $u$, and scalar products of $V$-valued functions instead of products of real-valued ones. It follows that the gradient of area ${ }_{\mu}$ evaluated at $x$ indeed equals $\Delta_{\mathrm{sd}} x$.

\subsection{Mean curvature of extrinsically flat surfaces}

We show that the mean curvature normal of a regular semidiscrete surface $x$ vanishes, if that surface is embedded in a 2-dimensional plane. Here, embeddedness means injectivity of the extended surface $x(s, t)$. Besides constituting a sanity check on our definitions, this fact is of importance later when we show the "linear precision" property of the semidiscrete Laplacian.

Lemma 1 If the regular semidiscrete surface $x: U \rightarrow V$ is embedded in a 2dimensional plane, then its mean curvature normal $\mathbf{H}_{\mathrm{sd}}$ vanishes.

Proof The general idea of the proof is to show that $\left\|\mathbf{H}_{\mathrm{sd}}\right\|_{L^{2}(x, V)}=0$ by constructing a variation whose derivative equals $\mathbf{H}_{\mathrm{sd}}$. This can be done in the following way. Choose a smooth function $v: U \rightarrow \mathbb{R}$ with compact support contained in $U^{\text {inn }}$. Then

$$
x_{\xi}(k, t):=x(k, t)+\xi v(k, t)^{2} \mathbf{H}_{\mathrm{sd}}(k, t)
$$

is a well-defined 1-parameter variation of $x$ with velocity $\dot{x}=v^{2} \mathbf{H}_{\mathrm{sd}}$.

Now, let $\Pi$ denote the plane containing the surface $x$ and assume without loss of generality that $0 \in \Pi$, so $\Pi$ is a linear subspace and therefore $\delta x, x^{\prime} \in \Pi$. It follows from Thm. 1 and Thm. 2 that $\mathbf{H}_{\mathrm{sd}}(k, t) \in \Pi$, and consequently, $x_{\xi}(k, t) \in \Pi$. Since $\operatorname{dim} \Pi=2$, we can express the above-mentioned area in terms of an appropriate determinant form $|\cdot, \cdot|$ :

$$
\begin{aligned}
\sqrt{\operatorname{det} \mathrm{I}_{\xi}(s, t)}= & \left|\delta x_{\xi}(s, t), x_{\xi}^{\prime}(s, t)\right|=(1-s+k)\left|\delta x_{\xi}(k, t), x_{\xi}^{\prime}(k, t)\right| \\
& +(s-k)\left|\delta x_{\xi}(k, t), x_{\xi}^{\prime}(k+1, t)\right|, \quad \text { for } s \in[k, k+1], t \text { fixed. }
\end{aligned}
$$


By Eq. 4 , integrating $\sqrt{\operatorname{det}_{\xi}}$ over $[k, k+1]$ w.r.t. $d \mu(s)$ is the same as integrating w.r.t. Lebesgue measure. Thus, $\operatorname{area}_{\mu}\left(\left.x_{\xi}\right|_{B}\right)$ equals the unsigned Euclidean area. Since the variation $x_{\xi}$ leaves the boundary of the surface unchanged, area ${ }_{\mu}\left(\left.x_{\xi}\right|_{B}\right)$ does not depend on $\xi$, and we get

$$
\begin{aligned}
\left\|v \mathbf{H}_{\mathrm{sd}}\right\|_{L^{2}(x, V)}^{2} & =\left\langle\mathbf{H}_{\mathrm{sd}}, v^{2} \mathbf{H}_{\mathrm{sd}}\right\rangle_{L^{2}(x, V)}=-\frac{1}{2}\langle\nabla \operatorname{area}(x), \dot{x}\rangle_{L^{2}(x, V)} \\
& =-\left.\frac{1}{2} \frac{d}{d \xi} \operatorname{area}\left(\left.x_{\xi}\right|_{B}\right)\right|_{\xi=0}=0 .
\end{aligned}
$$

We conclude that $v \mathbf{H}_{\text {sd }}$ vanishes for all $v$, i.e., $\mathbf{H}_{\text {sd }}=$ const. $=0$.

\section{Properties of the semidiscrete Laplacian}

The classical Laplace operator enjoys several properties like linear precision, symmetry, positive semidefiniteness, and an associated maximum principle for harmonic functions. It is natural to ask if they carry over to the purely discrete or semidiscrete cases (for triangle meshes, these core properties turn out to be incompatible for Laplacians whose definition involves the 1-ring neighbourhood of individual vertices; see [19]). We start by investigating the kernel of the Laplacian. Surely it contains the constant functions. As to linear functions, we have the following result:

Lemma 2 For a regular semidiscrete surface $x$ and its corresponding Laplacian $\Delta_{\mathrm{sd}}$ and mean curvature normal field $\mathbf{H}_{\mathrm{sd}}$, the following statements are equivalent:

(a) All functions $u(k, t)=L(x(k, t))$ with $L: V \rightarrow \mathbb{R}$ linear are contained in the kernel of $\Delta_{\text {sd. }}$.

(b) $x$ is harmonic, i.e., $\Delta_{\mathrm{sd}} x=$ const. $=0$.

(c) $x$ is a minimal surface, i.e., $\mathbf{H}_{\mathrm{sd}}=$ const. $=0$.

Proof The equivalence of (b) and (c) is Theorem 2. Since the coordinate components of $x$ are linear functions of $x$, (a) implies (b). Conversely, any linear function is a linear combination of coordinate functions and a constant, so (b) implies (a).

Corollary 1 The semidiscrete Laplacian enjoys the linear precision property, i.e., for a regular semidiscrete surface embedded in a 2-dimensional plane, all linear functions are contained in the kernel of the Laplacian.

Proof Combine Lemmas 1 and 2.

We show that our semidiscrete Laplacian is symmetric and positive semidefinite in the $L^{2}$ sense, in a way analogous to the well known Laplace-Beltrami operator (see, e.g. [15]). This follows directly from the variational definition of the Laplacian. 
Lemma 3 The semidiscrete Laplace operator $\Delta_{\mathrm{sd}}$ is symmetric and positive semidefinite. More precisely, for semidiscrete functions $u$ and $v$, with compact support contained in $U^{\text {inn }}$, we have

$$
\left\langle\Delta_{\mathrm{sd}} u, v\right\rangle_{L^{2}(x)}=\left\langle u, \Delta_{\mathrm{sd}} v\right\rangle_{L^{2}(x)} \quad \text { and } \quad\left\langle\Delta_{\mathrm{sd}} u, u\right\rangle_{L^{2}(x)}=2 E_{\mu}(u) \geq 0 .
$$

Proof We use the quadratic form $\mathcal{E}_{\mu}$ corresponding to the Dirichlet energy (see Definition 3) and compute $\left\langle\Delta_{\mathrm{sd}} u, v\right\rangle_{L^{2}(x)}=\left\langle\nabla E_{\mu}(u), v\right\rangle_{L^{2}(x)}=\left.\frac{d}{d \xi} E_{\mu}(u+\xi v)\right|_{\xi=0}$ $=\left.\frac{d}{d \xi}\left(\mathcal{E}_{\mu}(u, u)+2 \xi \mathcal{E}_{\mu}(u, v)+\xi^{2} \mathcal{E}_{\mu}(v, v)\right)\right|_{\xi=0}=2 \mathcal{E}_{\mu}(u, v)$, where we have used the relations given in Definition 5. This implies symmetry and, for $u=v$, semidefiniteness.

Unfortunately, the maximum principle is not valid for the semidiscrete Laplacian, even for functions on very simple surfaces. This is in contrast to the smooth case, where the maximum principle holds in general; and it is also in contrast to the cotanLaplacian on triangle meshes (likewise found as gradient of the Dirichlet energy), where a maximum principle holds e.g. if all angles are acute. A counterexample is as follows.

Example 2 Here we construct a semidiscrete harmonic function $u$ with a maximum at the inner point $(0,0)$ of the semidiscrete surface $x(k, t):=(k, t)$. For this purpose we first derive a more explicit expression for the Laplacian $\Delta_{\mathrm{sd}} u$ of a semidiscrete function $u$ on $x$. We extend $x$ to $x(s, t)=(s, t)$ and $u$ to the piecewise-linear function $u(s, t)=\sum_{(k, t) \in \mathbb{Z} \times \mathbb{R}} \varphi(s-k) u(k, t)$. Then $\mathrm{I}=\operatorname{diag}(1,1)$, so by the assumptions 4, we get

$$
\begin{aligned}
\mathbf{a}(k, t) & =\int_{[k-1, k] \sqcup[k, k+1]} \varphi(s-k) d \mu(s)=1, \\
\mathbf{b}(k, t) & =-\int_{[k-1, k]} \delta u(s, t) d \mu(s)=-\delta u(k-1, t)=u(k, t)-u(k-1, t), \\
\mathbf{c}(k, t) & =-\int_{[k-1, k] \sqcup[k, k+1]} \varphi(s-k) u^{\prime}(s, t) d \mu(s) \\
& =-2 m_{2} u^{\prime}(k, t)-\left(\frac{1}{2}-m_{2}\right)\left(u^{\prime}(k-1, t)+u^{\prime}(k+1, t)\right),
\end{aligned}
$$

where $m_{2}=\int_{[0,1]} s^{2} d \mu_{0}$ is the second moment of the measure $\mu_{0}$. Hence, in this situation, the Laplacian of $u$ is given by

$$
\begin{aligned}
\Delta_{\mathrm{sd}} u(k, t)= & 2 u(k, t)-u(k-1, t)-u(k+1, t)-2 m_{2} u^{\prime \prime}(k, t)-\left(\frac{1}{2}-m_{2}\right) \\
& \times\left(u^{\prime \prime}(k-1, t)+u^{\prime \prime}(k+1, t)\right) .
\end{aligned}
$$

The harmonicity condition $\Delta_{\mathrm{sd}} u=0$ thus becomes a system of linear ODEs for the functions $t \mapsto u(k, t)$, where $k$ runs through the integers. Observe that the assumptions 4 imply $\frac{1}{4} \leq m_{2} \leq \frac{1}{2}$. The maximum principle obviously holds if $m_{2}=\frac{1}{2}$, which applies e.g. to the trapezoidal rule. Otherwise, for $m_{2}<\frac{1}{2}$, we can construct a harmonic function $u$ on $x$ with a maximum at $(0,0)$ as follows. Choosing $u(0, t):=-t^{2}$ and assuming symmetry $u( \pm 1, t):=\phi(t)$, we find $\phi(t)$ easily 
as $\phi(t)=1-t^{2}+\gamma_{1} \cos \left(\left(\frac{1}{2}-m_{2}\right)^{-1 / 2} t\right)+\gamma_{2} \sin \left(\left(\frac{1}{2}-m_{2}\right)^{-1 / 2} t\right)$. An appropriate choice of constants, e.g. $\gamma_{1}=-2, \gamma_{2}=0$, yields a function $u(k, t)$, which undoubtedly has a local maximum in $u(0,0)=0$. We have thus created a locally defined counterexample to the maximum principle. It can be turned into a globally defined example by constructing $u( \pm 2, t), u( \pm 3, t), \ldots$ such that overall $\Delta_{\mathrm{sd}} u=0$ : one has to iteratively solve linear ODEs.

\section{Pointwise convergence / consistency}

In this section we show that the semidiscrete Laplace operator converges pointwise to its smooth counterpart, as the semidiscrete surface converges to a smooth one. In the Finite Elements literature this kind of convergence is called consistency, while convergence would be reserved for the situation where the solutions of equations involving the semidiscrete Laplacian converge to solutions of equations which involve the continuous Laplacian.

More precisely, the situation in the following theorem is as follows. We fix a point $p$ on a regular surface $\mathcal{M}$, which is assumed to have a local parametrization $f$. Without loss of generality, $p=f(0,0)$. Next, we consider the semidiscrete surface

$$
x_{\varepsilon}:(k, t) \mapsto f(\varepsilon k, t), \quad \varepsilon>0,
$$

which obviously contains the point $p=x_{\varepsilon}(0,0)$ and is inscribed in the surface $\mathcal{M}$. Then we analyze the semidiscrete Laplace operator associated with $x_{\varepsilon}$ and its action on functions $u_{\varepsilon}$, and let $\varepsilon \rightarrow 0$.

Theorem 3 Consider a smooth regular surface $\mathcal{M}$ with parametrization $f$ and a real-valued function $u(s, t)$ which represents a function defined on the surface $\mathcal{M}$. Let $p=f(0,0)$.

Semidiscretize these objects by defining a semidiscrete surface $x_{\varepsilon}(k, t):=$ $f(\varepsilon k, t)$ and a semidiscrete function $u_{\varepsilon}(k, t):=u(\varepsilon k, t)$. Then the corresponding semidiscrete Laplace operator $\Delta_{\mathrm{sd}}^{\varepsilon}$ converges to the Laplace-Beltrami operator $\Delta_{\mathcal{M}}$ defined on $\mathcal{M}$ :

$$
f, u \in C^{2} \Longrightarrow\left(\Delta_{\mathrm{sd}}^{\varepsilon} u_{\varepsilon}\right)(0,0)=\left(\Delta_{\mathcal{M}} u\right)(p)+o(1), \quad \text { as } \varepsilon \rightarrow 0 .
$$

In case the measure $\mu_{0}$ is symmetric in the sense of Eq. 5, convergence is improved:

$$
\begin{aligned}
& f, u \in C^{3} \Longrightarrow\left(\Delta_{\mathrm{sd}}^{\varepsilon} u_{\varepsilon}\right)(0,0)=\left(\Delta_{\mathcal{M}} u\right)(p)+\mathrm{o}(\varepsilon), \quad \text { as } \varepsilon \rightarrow 0, \\
& f, u \in C^{4} \Longrightarrow\left(\Delta_{\mathrm{sd}}^{\varepsilon} u_{\varepsilon}\right)(0,0)=\left(\Delta_{\mathcal{M}} u\right)(p)+\mathcal{O}\left(\varepsilon^{2}\right), \quad \text { as } \varepsilon \rightarrow 0 .
\end{aligned}
$$

Theorem 2 immediately implies a convergence statement concerning mean curvature:

Corollary 2 In the situation of Theorem 3, the semidiscrete mean curvature normal $\mathbf{H}_{\mathrm{sd}}^{\varepsilon}$ on $x_{\varepsilon}$ converges pointwise to its smooth counterpart (with the rate of convergence depending on the smoothness of the parametrization $f$ ). 
Proof of Theorem 3 We first set up some notation. For differentiation with respect to $s$ and $t$ we use the notation $\partial_{1}$ and $\partial_{2}$, respectively. The coefficients of the first fundamental form are denoted by $g_{i j}:=\left\langle\partial_{i} f, \partial_{j} f\right\rangle$. Their determinant is denoted by $\operatorname{det} \mathrm{I}=g_{11} g_{22}-g_{12}^{2}$. We also use the symbols $\rho_{i j k}:=\left\langle\partial_{i} f, \partial_{j k} f\right\rangle$.

- Step 1: Overview of the proof.

In local coordinates, the Laplacian is expressed as

$$
\begin{aligned}
\Delta_{\mathcal{M}} u & =\frac{1}{\mathbf{a}^{\text {cont }}}\left(\partial_{1} \mathbf{b}^{\text {cont }}+\partial_{2} \mathbf{c}^{\text {cont }}\right), \quad \text { where } \\
\mathbf{a}^{\text {cont }} & =\sqrt{\operatorname{det} \mathrm{I}}, \quad \mathbf{b}^{\text {cont }}=\frac{g_{12} \partial_{2} u-g_{22} \partial_{1} u}{\sqrt{\operatorname{det} \mathrm{I}}}, \quad \mathbf{c}^{\text {cont }}=\frac{g_{12} \partial_{1} u-g_{11} \partial_{2} u}{\sqrt{\operatorname{det} \mathrm{I}}}
\end{aligned}
$$

(see e.g. [15, p. 18]). On the other hand, the semidiscrete Laplacian $\Delta_{\text {sd }}^{\varepsilon}$ associated with $x_{\varepsilon}$ is computed, using notation $\mathbf{a}_{\varepsilon}, \mathbf{b}_{\varepsilon}, \mathbf{c}_{\varepsilon}$ analogous to Thm. 1, as

$$
\Delta_{\mathrm{sd}}^{\varepsilon} u_{\varepsilon}(0,0)=\left.\frac{1}{\mathbf{a}_{\varepsilon}}\left(\delta \mathbf{b}_{\varepsilon}+\mathbf{c}_{\varepsilon}^{\prime}\right)\right|_{(0,0)} .
$$

We compute Taylor polynomials around $(0,0)$ for $x_{\varepsilon}( \pm 1,0)=f( \pm \varepsilon, 0)$ and $u_{\varepsilon}( \pm 1,0)=u( \pm \varepsilon, 0)$, and insert them into this formula. Long computations yield

$$
\frac{\mathbf{a}_{\varepsilon}(0,0)}{\varepsilon} \approx \mathbf{a}^{\text {cont }}(0,0), \quad \frac{\delta \mathbf{b}_{\varepsilon}(0,0)}{\varepsilon} \approx \partial_{1} \mathbf{b}^{\text {cont }}(0,0), \quad \frac{\mathbf{c}_{\varepsilon}^{\prime}(0,0)}{\varepsilon} \approx \partial_{2} \mathbf{c}^{\text {cont }}(0,0),
$$

where the $\approx$ symbol means equality up to $\mathcal{O}\left(\varepsilon^{2}\right)$ in the $C^{4}$ case, resp. o $(\varepsilon)$ in the $C^{3}$ case, resp. o(1) in the $C^{2}$ case. Having obtained these convergence rates, the proof is complete. It remains to perform the above-mentioned long computations.

- Step 2: Taylor expansion of $x_{\varepsilon}, u_{\varepsilon}$ and their derivatives. Note that

$$
x_{\varepsilon}(s, 0)=\sum_{k} \varphi(s-k) x_{\varepsilon}(k, 0)= \begin{cases}(1+s) f(0,0)-s f(-\varepsilon, 0), & \text { if } s \in[-1,0], \\ (1-s) f(0,0)+s f(\varepsilon, 0), & \text { if } s \in[0,1] .\end{cases}
$$

The expression for $u_{\varepsilon}$ in terms of $u( \pm \varepsilon, 0)$ is analogous. The Taylor polynomials of $f( \pm \varepsilon, 0)$ and its derivatives around $\varepsilon=0$ are in the $C^{4}$ case given by

$$
\begin{aligned}
f( \pm \varepsilon, 0) & =f(0,0) \pm \varepsilon \partial_{1} f(0,0)+\frac{\varepsilon^{2}}{2} \partial_{11} f(0,0) \pm \frac{\varepsilon^{3}}{6} \partial_{111} f(0,0)+\mathcal{O}\left(\varepsilon^{4}\right), \\
\partial_{2} f( \pm \varepsilon, 0) & =\partial_{2} f(0,0) \pm \varepsilon \partial_{12} f(0,0)+\frac{\varepsilon^{2}}{2} \partial_{112} f(0,0)+\mathcal{O}\left(\varepsilon^{3}\right) . \\
\partial_{22} f( \pm \varepsilon, 0) & =\partial_{22} f(0,0) \pm \varepsilon \partial_{122} f(0,0)+\mathcal{O}\left(\varepsilon^{2}\right) .
\end{aligned}
$$

The remainder terms $\mathcal{O}\left(\varepsilon^{j}\right)$ in the individual formulas have to be replaced by $\mathrm{o}\left(\varepsilon^{j-1}\right)$ in the $C^{3}$ case. In the $C^{2}$ case, the terms containing third order partial derivatives of $f$ have to be replaced by $\mathrm{o}\left(\varepsilon^{j-2}\right)$. There are analogous expressions for $u( \pm \varepsilon, 0)$ and its derivatives.

- Step 3: Taylor expansion of the area element. For sufficiently small $\varepsilon>0$, we consider the first fundamental form $\mathrm{I}_{\varepsilon}$ associated with the piecewise-ruled surface $x_{\varepsilon}$ and look at the quantity

$$
\alpha(\varepsilon, s)=\sqrt{\operatorname{det} \mathrm{I}_{\varepsilon}(s, 0)}, \quad s \in[-1,1] .
$$


Note that, for $s \in[0,1]$, det $\mathrm{I}_{\varepsilon}(s, 0)$ is the Gram determinant of vectors

$$
\delta x_{\varepsilon}(s, 0)=f(\varepsilon, 0)-f(0,0), x_{\varepsilon}^{\prime}(s, 0)=(1-s) \partial_{2} f(0,0)+s \partial_{2} f(\varepsilon, 0) .
$$

In the $C^{4}$ case, a simple computation and taking square roots by means of the binomial series yields

$$
\begin{aligned}
& \operatorname{det} \mathrm{I}_{\varepsilon}(s, 0)=\varepsilon^{2}\left(\alpha_{1}+\varepsilon\left(\alpha_{2}+s \alpha_{3}\right)+\varepsilon^{2} \alpha_{4}+\mathcal{O}\left(\varepsilon^{3}\right)\right), \quad \text { as } \varepsilon \rightarrow 0, \\
& \alpha_{1}=\left.\operatorname{det} \mathrm{I}\right|_{(0,0)}, \alpha_{2}=\left.\left(g_{22} \rho_{111}-g_{12} \rho_{211}\right)\right|_{(0,0)}, \alpha_{3}=\left.2\left(g_{11} \rho_{212}-g_{12} \rho_{112}\right)\right|_{(0,0)}, \\
& \alpha(\varepsilon, s)=|\varepsilon|\left(\alpha_{1}^{1 / 2}+\frac{\varepsilon}{2} \frac{\alpha_{2}+s \alpha_{3}}{\alpha_{1}^{1 / 2}}+\frac{\varepsilon^{2}}{8} \frac{4 \alpha_{1} \alpha_{4}-\left(\alpha_{2}+s \alpha_{3}\right)^{2}}{\alpha_{1}^{3 / 2}}+\mathcal{O}\left(\varepsilon^{3}\right)\right) .
\end{aligned}
$$

In the $C^{3}$ case, the remainder term is o $\left(\varepsilon^{2}\right)$, while in the $C^{2}$ case, the terms involving $\varepsilon^{2}$ have to be replaced by $\mathrm{o}(\varepsilon)$. For $s \in[-1,0]$, the situation is analogous.

- $\quad$ Step 4: The relation between $\mathbf{a}_{\varepsilon}$ and $\mathbf{a}^{\text {cont }}$. Our aim is to give a proof of $\frac{1}{\varepsilon} \mathbf{a}_{\varepsilon}(0,0)$ $\approx \mathbf{a}^{\text {cont }}(0,0)$, where the meaning of " $\approx$ " is equality up to an error term depending on the differentiability class of the objects involved. The relation $\alpha(\varepsilon,-s)=$ $\alpha(-\varepsilon, s)$ yields

$$
\begin{aligned}
\mathbf{a}_{\varepsilon}(0,0) & =\int_{[-1,0]}(1+s) \alpha(\varepsilon, s) d \mu(s)+\int_{[0,1]}(1-s) \alpha(\varepsilon, s) d \mu(s) \\
& =\int_{[0,1]} s \alpha(-\varepsilon, 1-s)+(1-s) \alpha(\varepsilon, s) d \mu(s) \\
& =|\varepsilon| \int_{[0,1]}\left(\alpha_{1}^{1 / 2}+\frac{\varepsilon}{2}(1-2 s) \alpha_{2} \alpha_{1}^{-1 / 2}+O\left(\varepsilon^{2}\right)\right) d \mu(s)
\end{aligned}
$$

in the $C^{3}$ and $C^{4}$ cases, and the same formula with remainder term o $(\varepsilon)$ in the $C^{2}$ case. Now Eq. 4 yields $\int_{[0,1]}(1-2 s) d \mu(s)=0$, so the result follows.

- Step 5: Relation between $\mathbf{b}_{\varepsilon}$ and $\mathbf{b}^{\text {cont }}$. With computations similar to those of the previous Step 4, it is not difficult to see that

$$
\delta \mathbf{b}_{\varepsilon}(0,0)=\mathbf{b}_{\varepsilon}(1,0)-\mathbf{b}_{\varepsilon}(0,0)=\int_{[0,1]}\left(\frac{\beta(-\varepsilon, 1-s)}{\alpha(-\varepsilon, 1-s)}+\frac{\beta(\varepsilon, s)}{\alpha(\varepsilon, s)}\right) d \mu_{0}(s),
$$

where $\beta(\varepsilon, s):=\left\langle\delta x_{\varepsilon}, x_{\varepsilon}^{\prime}\right\rangle u_{\varepsilon}^{\prime}-\left.\left\|x_{\varepsilon}^{\prime}\right\|^{2} \delta u_{\varepsilon}\right|_{(0,0)}$ is expressed as

$$
\begin{aligned}
\beta(\varepsilon, s)= & \left\langle f(\varepsilon, 0)-f(0,0),(1-s) \partial_{2} f(0,0)+s \partial_{2} f(\varepsilon, 0)\right\rangle \\
& \cdot\left((1-s) \partial_{2} u(0,0)+s \partial_{2} u(\varepsilon, 0)\right) \\
& -\left\|(1-s) \partial_{2} f(0,0)+s \partial_{2} f(\varepsilon, 0)\right\|^{2}(u(\varepsilon, 0)-u(0,0)), \text { for } s \in[0,1] .
\end{aligned}
$$

Note that $\beta(\varepsilon,-s)=-\beta(-\varepsilon, s)$ and $\alpha(\varepsilon,-s)=\alpha(-\varepsilon, s)$ for $s \in[0,1]$. Assuming symmetry of the measure $\mu_{0}$, this simplifies to

$$
\delta \mathbf{b}_{\varepsilon}(0,0)=\int_{[0,1]}\left(\frac{\beta(-\varepsilon, s)}{\alpha(-\varepsilon, s)}+\frac{\beta(\varepsilon, s)}{\alpha(\varepsilon, s)}\right) d \mu_{0}(s) .
$$


Inserting Taylor polynomials yields the expansion (for the $C^{3}$ case)

$$
\begin{aligned}
\beta(\varepsilon, s) & =\varepsilon \beta_{1}+\varepsilon^{2}\left(\beta_{2}+s \beta_{3}\right)+\varepsilon^{3} \beta_{4}+\mathrm{o}\left(\varepsilon^{3}\right), \quad \text { where } \\
\beta_{1} & =g_{12} \partial_{2} u-g_{22} \partial_{1} u\left|0,0, \quad \beta_{2}=\frac{1}{2}\left(\rho_{211} \partial_{2} u-g_{22} \partial_{11} u\right)\right|_{0,0}, \\
\beta_{3} & =\left.\left(\rho_{112} \partial_{2} u-2 \rho_{212} \partial_{1} u+g_{12} \partial_{12} u\right)\right|_{0,0} .
\end{aligned}
$$

This leads to

$$
\frac{\beta(-\varepsilon, s)}{\alpha(-\varepsilon, s)}+\frac{\beta(\varepsilon, s)}{\alpha(\varepsilon, s)}=\frac{\varepsilon^{3}\left(2\left(\beta_{2}+s \beta_{3}\right) \alpha_{1}^{1 / 2}-\beta_{1}\left(\alpha_{2}+s \alpha_{3}\right) \alpha_{1}^{-1 / 2}\right)+\mathrm{o}\left(\varepsilon^{4}\right)}{\varepsilon^{2} \alpha_{1}+\mathrm{o}\left(\varepsilon^{3}\right)} .
$$

Integration with respect to $d \mu(s)$ and substituting the definitions of $\alpha_{j}, \beta_{j}$ eventually yields

$$
\frac{\delta \mathbf{b}_{\varepsilon}(0,0)}{\varepsilon}=\frac{2 \beta_{2}+\beta_{3}}{\alpha_{1}^{1 / 2}}-\frac{\beta_{1}\left(\alpha_{2}+\frac{1}{2} \alpha_{3}\right)}{\alpha_{1}^{3 / 2}}+\mathrm{o}(\varepsilon)=\partial_{1} \mathbf{b}^{\mathrm{cont}}(0,0)+\mathrm{o}(\varepsilon) .
$$

In the $C^{4}$ case the remainder term is $\mathcal{O}\left(\varepsilon^{2}\right)$, whereas in the $C^{2}$ case, where symmetry of the measure is not required, the integral on the right hand side of Eq. 9 does not simplify as shown above, and we only get a remainder term of $o(1)$.

- Step 6: Computing the derivative of $\mathbf{c}(k, t)$. The following explicit formula, which is found by differentiating the definition of $\mathbf{c}(k, t)$, is needed later:

$$
\begin{aligned}
& \frac{d \mathbf{c}(k, t)}{d t}=\int_{[k-1, k] \sqcup[k, k+1]} \varphi(s-k) \frac{\partial}{\partial t}\left(\frac{\left\langle\delta x, x^{\prime}\right\rangle \delta u-\|\delta x\|^{2} u^{\prime}}{\operatorname{det} \mathrm{I}^{1 / 2}}\right) d \mu(s) \\
& =\int_{[k-1, k] \sqcup[k, k+1]} \varphi(s-k)\left(\frac{c^{*}(s, t)}{\operatorname{det} \mathrm{I}^{1 / 2}}-\frac{c^{* *}(s, t)}{\operatorname{det} \mathrm{I}^{3 / 2}}\right) d \mu(s), \quad \text { where } \\
& c^{*}:=\left(\left\langle\delta x^{\prime}, x^{\prime}\right\rangle+\left\langle\delta x, x^{\prime \prime}\right\rangle\right) \delta u+\left\langle\delta x, x^{\prime}\right\rangle \delta u^{\prime}-2\left\langle\delta x, \delta x^{\prime}\right\rangle u^{\prime}-\|\delta x\|^{2} u^{\prime \prime}, \\
& c^{* *}:=\left(\left\langle\delta x, x^{\prime}\right\rangle \delta u-\|\delta x\|^{2} u^{\prime}\right)\left[\left\langle x^{\prime}, x^{\prime \prime}\right\rangle\|\delta x\|^{2}-\left(\left\langle\delta x^{\prime}, x^{\prime}\right\rangle+\left\langle\delta x, x^{\prime \prime}\right\rangle\right)\left\langle\delta x, x^{\prime}\right\rangle\right. \\
& \left.+\left\langle\delta x, \delta x^{\prime}\right\rangle\left\|x^{\prime}\right\|^{2}\right] \text {. }
\end{aligned}
$$

- Step 7: Relation between $\mathbf{c}_{\varepsilon}$ and $\mathbf{c}^{\text {cont }}$. We use the notation of Step 6 to introduce the symbols $\gamma^{*}(\varepsilon, s), \gamma^{* *}(\varepsilon, s)$, which arise from the functions $c^{*}, c^{* *}$, resp., by substituting $x_{\varepsilon}$ for $x$ and $u_{\varepsilon}$ for $u$, and letting $t=0$. Note that $\gamma^{*}(\varepsilon,-s)=$ $\gamma^{*}(-\varepsilon, s)$ and the same for $\gamma^{* *}$, for $s \in[0,1]$. With a computation similar to Step 4 , it is easy to see that $\mathbf{c}_{\varepsilon}^{\prime}(0,0)$ is expressed as

$$
\int_{[0,1]} s \frac{\gamma^{*}(-\varepsilon, 1-s)}{\alpha(-\varepsilon, 1-s)}+(1-s) \frac{\gamma^{*}(\varepsilon, s)}{\alpha(\varepsilon, s)}-s \frac{\gamma^{* *}(-\varepsilon, 1-s)}{\alpha(-\varepsilon, 1-s)^{3}}-(1-s) \frac{\gamma^{* *}(\varepsilon, s)}{\alpha(\varepsilon, s)^{3}} d \mu_{0}(s) .
$$

Assuming symmetry of the measure $\mu_{0}$, this expression simplifies to

$$
\int_{[0,1]}(1-s)\left(\frac{\gamma^{*}(-\varepsilon, s)}{\alpha(-\varepsilon, s)}+\frac{\gamma^{*}(\varepsilon, s)}{\alpha(\varepsilon, s)}\right)-(1-s)\left(\frac{\gamma^{* *}(-\varepsilon, s)}{\alpha(-\varepsilon, s)^{3}}+\frac{\gamma^{* *}(\varepsilon, s)}{\alpha(\varepsilon, s)^{3}}\right) d \mu_{0}(s) .
$$


In the same manner as before we get the expansions

$$
\begin{aligned}
\gamma^{*}(\varepsilon, s) & =\varepsilon^{2} \gamma_{1}^{*}+\varepsilon^{3}\left(\gamma_{2}^{*}+s \gamma_{3}^{*}\right)+o\left(\varepsilon^{3}\right), \\
\gamma^{* *}(\varepsilon, s) & =\varepsilon^{4} \gamma_{1}^{* *}+\varepsilon^{5}\left(\gamma_{2}^{* *}+s \gamma_{3}^{* *}\right)+o\left(\varepsilon^{5}\right),
\end{aligned}
$$

where

$$
\begin{aligned}
\gamma_{1}^{*} & =\left.\left(\left(\rho_{122}+\rho_{212}\right) \partial_{1} u+g_{12} \partial_{12} u-2 \rho_{112} \partial_{2} u-g_{11} \partial_{22} u\right)\right|_{(0,0)}, \\
\gamma_{1}^{* *} & =\left.\left(g_{12} \partial_{1} u-g_{11} \partial_{2} u\right)\left(\rho_{112} g_{22}+\rho_{222} g_{11}-\left(\rho_{212}+\rho_{122}\right) g_{12}\right)\right|_{(0,0)} .
\end{aligned}
$$

This leads to

$$
\begin{aligned}
\frac{\gamma^{*}(-\varepsilon, s)}{\alpha(-\varepsilon, s)}+\frac{\gamma^{*}(\varepsilon, s)}{\alpha(\varepsilon, s)}=\frac{1}{\varepsilon^{2} \alpha_{1}+\mathrm{o}\left(\varepsilon^{3}\right)}\left(\varepsilon^{3} 2 \gamma_{1}^{*} \alpha_{1}^{1 / 2}+\mathrm{o}\left(\varepsilon^{4}\right)\right)=\varepsilon \frac{2 \gamma_{1}^{*}}{\alpha_{1}^{1 / 2}}+\mathrm{o}\left(\varepsilon^{2}\right), \\
\frac{\gamma^{* *}(-\varepsilon, s)}{\alpha(-\varepsilon, s)^{3}}+\frac{\gamma^{* *}(\varepsilon, s)}{\alpha(\varepsilon, s)^{3}}=\frac{1}{\varepsilon^{6} \alpha_{1}^{3}+o\left(\varepsilon^{7}\right)}\left(\varepsilon^{7} 2 \gamma_{1}^{* *} \alpha_{1}^{3 / 2}+\mathrm{o}\left(\varepsilon^{8}\right)\right)=\varepsilon \frac{2 \gamma_{1}^{* *}}{\alpha_{1}^{3 / 2}}+\mathrm{o}\left(\varepsilon^{2}\right) .
\end{aligned}
$$

Using property (4) of the measure $\mu_{0}$ for integration, and substituting the definitions of $\alpha_{1}, \gamma_{1}^{*}, \gamma_{1}^{* *}$, one eventually gets

$$
\frac{\mathbf{c}_{\varepsilon}^{\prime}(0,0)}{\varepsilon}=\frac{\gamma_{1}^{*}}{\alpha_{1}^{1 / 2}}-\frac{\gamma_{1}^{* *}}{\alpha_{1}^{3 / 2}}+\mathrm{o}(\varepsilon)=\partial_{2} \mathbf{c}^{\mathrm{cont}}(0,0)+o(\varepsilon) .
$$

This result applies to the $C^{3}$ case. In the $C^{4}$ case, one more term in the Taylor polynomials becomes available, and the remainder term in the formula above becomes $\mathcal{O}\left(\varepsilon^{2}\right)$ instead of $o(\varepsilon)$. In the $C^{2}$ case, where symmetry of the measure $\mu_{0}$ is not required, we only get $\mathrm{o}(1)$ (the details are omitted). The estimates of steps 4,5 , and 7 together conclude the proof.

Acknowledgments This research was supported by the DFG Collaborative Research Center, TRR 109, "Discretization in Geometry and Dynamics" and the Doctoral Program "Discrete Mathematics" through grants I 706-N26 and W1230 of the Austrian Science Fund (FWF).

Open Access This article is distributed under the terms of the Creative Commons Attribution 4.0 International License (http://creativecommons.org/licenses/by/4.0/), which permits unrestricted use, distribution, and reproduction in any medium, provided you give appropriate credit to the original author(s) and the source, provide a link to the Creative Commons license, and indicate if changes were made.

\section{References}

1. Alexa, M., Wardetzky, M.: Discrete Laplacians on general polygonal meshes. ACM Trans. Graph. 30(4), 102:1-102:10 (2011)

2. Belkin, M., Sun, J., Wang, Y.: Discrete Laplace Operator on Meshed Surfaces. In: Proc. of the 24th Annual Symposium on Computational Geometry, pp. 278-287. ACM (2008)

3. Bobenko, A., Springborn, B.: A discrete Laplace-Beltrami operator for simplicial surfaces. Discret. Comput. Geom. 38(4), 740-756 (2007)

4. Bobenko, A.I., Suris, Y.B.: Discrete differential geometry. American Math. Soc. (2008)

5. Carl, W.: A Laplace operator on semi-discrete surfaces. Found. Comput. Math. (2015)

6. do Carmo, M.P.: Differential geometry of curves and surfaces. Prentice-Hall (1976) 
7. Colding, T.H., Minicozzi, W.P.: A course in minimal surfaces. American Math. Soc. (2011)

8. Dziuk, G.: Finite Elements for the Beltrami Operator on Arbitrary Surfaces. In: S. Hildebrandt, R. Leis (eds.) Partial differential equations and calculus of variations, pp. 142-155. Springer (1988)

9. Jost, J.: Riemannian Geometry and Geometric Analysis, 6th edn. Springer (2011)

10. Karpenkov, O., Wallner, J.: On offsets and curvatures for discrete and semidiscrete surfaces. Beitr. Algebra Geom. 55(1), 207-228 (2014)

11. Liu, D., Xu, G., Zhang, Q.: A discrete scheme of Laplace-Beltrami operator and its convergence over quadrilateral meshes. Comput. Math. Appl. 55(6), 1081-1093 (2008)

12. Pinkall, U., Polthier, K.: Computing discrete minimal surfaces and their conjugates. Exp. Math. 2(1), 15-36 (1993)

13. Pottmann, H., Schiftner, A., Bo, P., Schmiedhofer, H., Wang, W., Baldassini, N., Wallner, J.: Freeform surfaces from single curved panels. ACM Trans. Graph. 27(3), 76:1-76:10 (2008)

14. Pottmann, H., Wallner, J.: Computational line geometry. Springer (2001)

15. Rosenberg, S.: The Laplacian on a Riemannian manifold. Cambridge Univ. Press (1997)

16. Sun, J., Ovsjanikov, M., Guibas, L.J.: A concise and provably informative multi-scale signature based on heat diffusion. Comput. Graph. Forum. 28(5), 1383-1392 (2009)

17. Wallner, J.: On the semidiscrete differential geometry of A-surfaces and K-surfaces. J. Geom. 103, $161-176(2012)$

18. Wardetzky, M.: Convergence of the Cotangent Formula: An Overview. In: Discrete Differential Geometry, Oberwolfach Semin., Vol. 38, pp. 275-286. Birkhäuser (2008)

19. Wardetzky, M., Mathur, S., Kälberer, F., Grinspun, E.: Discrete Laplace Operators: No Free Lunch. In: Geometry Processing 2007, pp. 33-37. Eurographics Association (2007)

20. Wu, J.Y., Chi, M.H., Chen, S.G.: Convergent discrete Laplace-Beltrami operators over surfaces (2010). arXiv: 1004.3486

21. Xu, G.: Discrete Laplace-Beltrami operators and their convergence. Comput. Aided Geom. Des. 21(8), 767-784 (2004) 